Bangladesh Rice J. 20 (1) : 11-16, 2016

\title{
Estimating Area and Production of Rice under Different Crop-cut Methods in Bangladesh
}

\author{
M S Kabir1*, D N R Paul², M I Hossain ${ }^{3}$ and N M F Rahman
}

\begin{abstract}
Crop-cut is a widely used and well accepted procedure for estimating rice yield. This yield is then multiplied by the area planted under rice cultivation to determine rice production of the country. In Bangladesh, crop-cut is used to estimate transplanted rice yield. Department of Agricultural Extension (DAE) and Bangladesh Bureau of Statistics (BBS) as the public agencies provide estimate on area and production of rice but they use different methods of crop-cut. The estimates of these two agencies on area and production of rice are different posing a question to the users on the reliability of the estimates. An attempt has been made with the objective to formulate a protocol that provides reliable and unique estimates on rice area and production. It is suggested to make at least three cuts in each plot to minimize the chance of over or under estimation of the whole plot yield and the radius of the circle should be $178.5 \mathrm{~cm}$ so that the cut area becomes $10 \mathrm{~m}^{2}$ in order to easy conversion of the crop-cut yield to $t$ ha-1.
\end{abstract}

Key words: Crop-cut, BBS, DAE, rice, area and production

\section{INTRODUCTION}

Rice is the staple food crop and occupies about $80 \%$ of the country's total cropped area. To assess the total rice production, it is essential to estimate the accurate yield and production of rice. Real estimation is needed for future projection of rice production in relation to population growth, which is important at national level. Crop cutting in the real sense is a statistical survey method to estimate yield of crop in a population through cutting a crop at small parts of fields. In Bangladesh, crop area figures are compiled on the basis of complete enumeration while the crop yield is estimated on the basis of sample survey approach and crop cut is the most important method of this approach. A series of studies were carried out by the statistician and a scientific technique was developed for conducting the crop cutting experiments to obtain the reliable yield rates (Mahalanobis,
1945; Sukhatme and Panse, 1951; Murthy, 1997). Also, Department of Agricultural Extension (DAE, 1990) and Bangladesh Bureau of Statistics (BBS, 1982) adopted two different methods of crop-cut for yield estimation. DAE estimates yield from a rectangular area of 10 square meters and BBS estimates from a circular area of radius $5^{\prime} 7 \frac{1}{2}=9.248 \mathrm{~m}^{2}$. At least one of the methods may have some limitations as their estimates differ by a substantial degree. So, for the different methods of crop-cut, users and stakeholders often confused about these two organizations method and which one is better for rice yield estimates. Later, BBS (2013) tried to uniform BBS-DAE crop cutting method through Harmonization and Dissemination of Unified Agricultural Production Statistics (HDUAPS) project. Recently, BBS (2014) published a report on crop cutting experiments in Bangladesh. Yet still, in any farmers field the researcher/agriculture

\footnotetext{
${ }^{1}$ Director (Administration and Common Service), BRRI; ${ }^{2}$ Former Chief Scientific Officer and Head, ${ }^{3 P r i n c i p l e ~ S c i e n t i f i c ~}$ Officer and Head, ${ }^{4}$ Senior Scientific Officer, Agricultural Statistics Division, BRRI, Gazipur, Bangladesh. *Corresponding author's E-mail: kabir.stat@gmail.com
} 
extension worker, stakeholders has to face the problem for assessing the rice yield through crop-cut method. Because, the researchers, extension personnel and stakeholders have been facing the problem about BBS unified crop cut method. To avoid these sorts of confusions among government agencies a consensus among ministry of Planning, Ministry of Agriculture and Ministry of Food, BRRI statisticians proposed a modified procedure to estimates the yield of rice through crop cut. The objective of this study is to estimate rice yield through crop-cuts at farmers' field and to formulate a protocol that provides suitable estimates of area and production of rice in Bangladesh.

\section{MATERIALS AND METHOD}

The technique of crop cut experiment (CCE) has various steps. There is certain equipment/material required for conducting the crop cutting experiments. These are:

- Measuring tape as per requirement (30 or 50 meter)

- Weighing balance as per requirement (Beam of spring balance)

- Set of weights $(1,2,5,10,20,50,100,200$, $500 ; 1,2$ and $5 \mathrm{~kg}$ )

- String or rope (30 meter)

- Four pegs

- The Hessian Cloth: is a coarsely woven fabric usually made from vegetable fibers and jute

- Cloth bags for keeping the produce for crying

- Two strong water proof bags (one for keeping crop cutting equipment's and other for keeping schedules and papers etc.)

There are various steps involved in the conduct of crop cutting experiments. These steps are:
- Selection of field (including size and shape of the CCE plot) where crop cutting experiment is to be carried out,

- Locating and marking of the experimental plot of a given size in the selected field,

- Harvesting of the CCE plot,

- Threshing of crop harvested from the CCE plot,

- Winnowing and weighing of the produce obtained from the CCE plot,

- Drying of produce, if it contains moisture, and

- Weight of the dry produce.

The study was conducted at Kapasia upazila of Gazipur in Boro season 2007. The crop-cut was done in a farmer's field in Boro. Farmers were selected randomly. At maturity, crop was cut and yield was estimated following standard procedure. Weight was taken and moisture was adjusted. The present crop-cut methods include the followings:

\section{Method A followed by DAE}

For each variety, DAE takes three cuts each of size $5-\times-2 m$, one from good crop, one from intermediate crop and the other from a bad crop in each block. The crop condition (good, intermediate and bad) is judged by visual inspection of crop stand, which is very much subjective and is likely to vary from person to person. Rice area in each season planted to different varieties is determined through farmer's interview by the Sub-Assistant Agriculture Officers (former Block Supervisors). At the block level, the moisture content of crop-cut paddy is used as per directives of the upazila agriculture office. The directives are based on some sample crop-cuts and measurement of moisture content using moisture meter. DAE also uses BBS method of crop-cut where BBS personnel join DAE in crop-cut. 


\section{Method B followed by BBS}

BBS (BBS, 1982), on the other hand, uses a three-fold instrument (Fig. 1) where crop-cuts are done in three circular areas (Fig. 2). Crops are harvested separately from each shaded area starting from the middle one and weighed. All harvests are then mixed and a sample of one $\mathrm{kg}$ of paddy from the harvest is brought to the office and dried for 10 days under room condition and weighed. The sample is then weighed at every evening until the weight becomes stable. The dry weight of paddy for each shaded area is recorded by adjusting the loss in weight of the sample. The three adjusted weights are then added together that gives the dry weight of paddy for the circular area of radius $57 \frac{1}{2}^{\prime \prime} \approx 9.248 \mathrm{~m}^{2}$ and converted to $\mathrm{tha}^{-1}$ that represents yield of the plot selected for crop-cut. However, in cases where DAE and BBS jointly do the cropcut, the moisture content is measured using moisture meter where available.

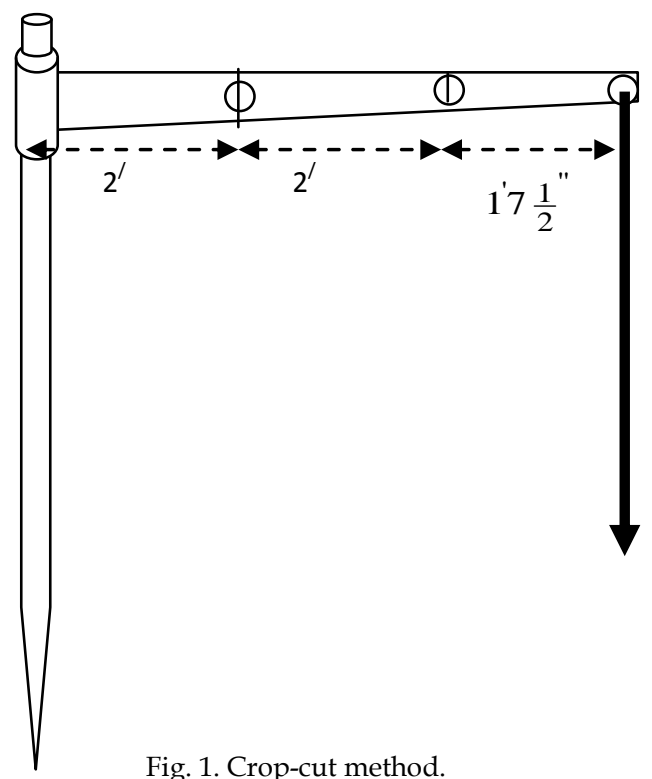

Fig. 1. Crop-cut method.

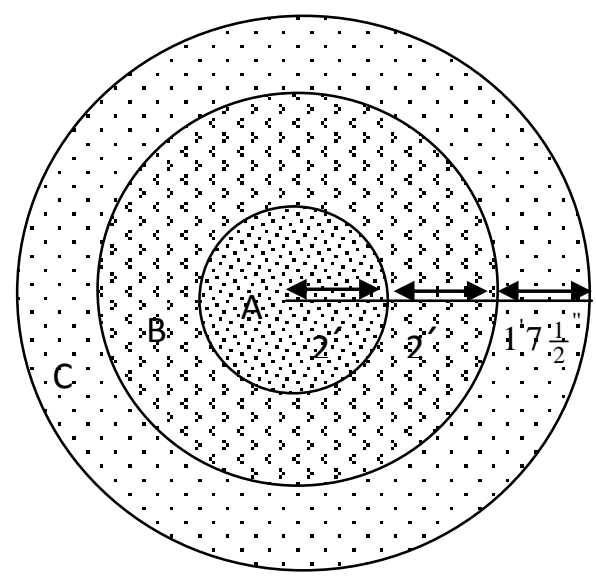

Fig. 2. Crop-cut method

\section{Comparison of yield estimates using DAE} and BBS procedures

Procedures followed by DAE (Method A) and BBS (Method B) were compared through actual crop-cut taken in farmer's field. Cropcut was done in a farmer's field in Boro 2007 at Kapasia upazila of Gazipur district. The variety was BRRI dhan29. Before crop-cut, an area of $300 \mathrm{~m}^{2}(20-x-15) \mathrm{m}$ was demarcated in the field leaving one meter from each levy as boarder. The area was then divided into 12 small areas each with (5-x-5) m size (Fig. 3). Twelve crop-cuts, six following method A and six following method $\mathrm{B}$ were done within $300 \mathrm{~m}^{2}$ area. The assignment of 12 cuts to 12 small areas was done at random. Figure 3 shows the position of each cut with their sequence number.

For method A, three cut areas were laid out with length of the cuts in the north-south direction and three with length of the cuts in the east-west direction and the position of each cut was at the middle of each small area. Crops were harvested separately from each cut area, threshed, cleaned, weighed and moisture content was measured using a moisture meter.

For method B, the centre of the circle was positioned at the middle of each small area. The harvests were then threshed, cleaned, weighed and moisture content was measured 


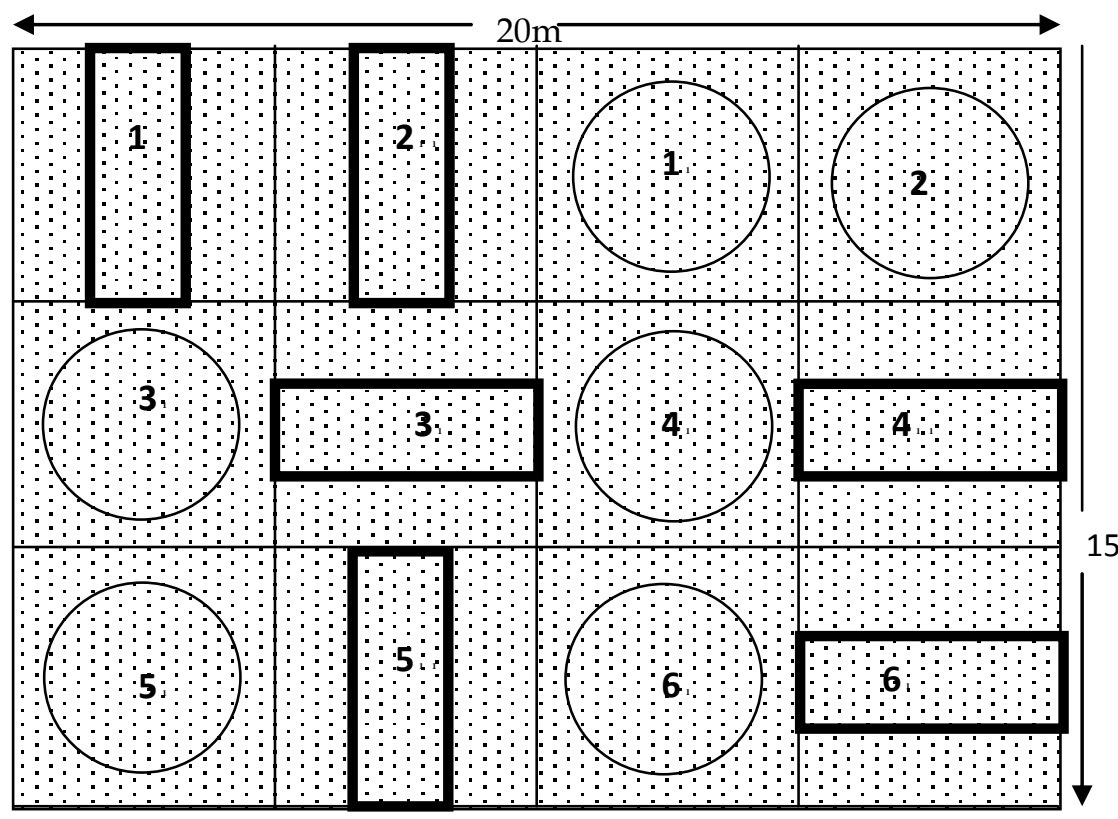

Fig. 3. Position of 12 different cuts with their sequence number (a rectangles represents a rectangular cut of size (5-x-2) $\mathrm{m}$ and a circle represents a circular cut of an area of $9.248 \mathrm{~m}^{2}$ )

separately for each portion of a crop-cut (three shaded area of Fig. 2). In case the pointer goes through a hill, the tillers falling within the circle were harvested. If the pointer passes through several hills, alternate hills were included in the crop-cut.

The yield estimates using different methods and that for the whole plot $\left(300 \mathrm{~m}^{2}\right)$ were obtained as follows:

Method A. The average of three measures of moistures was considered to be the moisture content of the fresh harvest for each crop-cut and adjusted at $14 \%$ moisture content. The adjusted weight was then converted to $t \mathrm{ha}^{-1}$. The formula for adjusting fresh weight at $14 \%$ moisture content is given by

Adjusted weight at $14 \%$ moisture content $=\frac{w(00-M C)}{86}$ where, $\mathrm{w}=$ fresh weight, $\mathrm{MC}=$ moisture content

Method B. The average of three measures of moisture content was considered to be the moisture content of the fresh harvest for each portion of a circular cut and adjusted at $14 \%$ moisture content. The adjusted weight was then added together and converted to $t \mathrm{ha}^{-1}$ that gives the weight of paddy for a circular area of radius $57 \frac{1}{2}^{\prime \prime}$.

Method C (proposed). Yield was estimated using a modified procedure where all hills within the circular area of radius $57 \frac{1}{2}$ were considered to be harvested through a single cut instead of harvesting crops in three portions (Fig. 2) under method B. For the present exercise, the fresh weight of paddy obtained from three portions of a circular cut under method $\mathrm{B}$ were added and the average moisture content of nine measures of moisture content was used to estimate the rice yield. The fresh weight (total of three portions of a circular cut) was then adjusted to $14 \%$ moisture content and converted to $t \mathrm{ha}^{-1}$.

Estimation of whole plot yield. The rest of the area $\left(184.5 \mathrm{~m}^{2}\right)$ that remained after 12 crop-cuts was harvested separately, threshed, cleaned and weighed. The moisture content 
was the average of the moisture content measured from three samples from the lot. The fresh harvest of the remaining area was then adjusted to $14 \%$ moisture content and converted to $t \mathrm{ha}^{-1}$. The weight of the whole plot was obtained as the total weight of remaining area and the weights of all cuts done using methods $\mathrm{A}$ and $\mathrm{B}$.

\section{Computation of precision and relative} efficiency of the estimates

(a) The mean yield of six cuts under each crop-cut method is given by

$\bar{y}=\frac{\sum_{i=1}^{6} y_{i}}{6}$, where $\mathrm{y}_{\mathrm{i}}$ is the estimate of yield for $\mathrm{i}^{\text {th }}$ cut

(b) The variance $(\mathrm{V})$ of the estimates for each crop-cut method is obtained as

$V($ Yield $)=\frac{\sum_{i=1}^{6}\left(\mathbf{Q}_{i}-\mu^{3}\right.}{6}$, where $\mathrm{y}_{\mathrm{i}}$ is the estimate of yield for $\mathrm{i}^{\text {th }}$ cut and $\mu$ is the true or the whole plot yield.

(c) The relative efficiency (RE) of a method over that of method $\mathrm{A}$ is measured as

$\operatorname{RE}($ Method $\mathrm{B})=\frac{V \text { Method } A}{V \text { Method } B)} \leq \times 100$ and

$\operatorname{RE}($ Method $C)=\frac{V \text { Method } A}{V \text { Method } C)}<100$

\section{RESULTS AND DISCUSSION}

Table 1 presents the estimated yields following different crop-cut methods. Results show a large variation in the estimate of yield using method A compared to that using methods $B$ and $C$. The high variability in the estimate for the method $\mathrm{A}$ is due to overall fertility gradient of the field from east to west. This is evident from the fact that the estimates for the cuts with length in the north-south direction are higher than those for the cuts with length in the east-west direction. In general, yield estimated using method $\mathrm{A}$ is found to be higher than the yield estimated by circular methods. In case of method $\mathrm{A}$, the percent deviation of crop-cut yield from the whole plot yield ranges $2-14 \%$, which is less than $2 \%$ in case of methods B and C. It is seen that average of crop-cut yields estimated using either of the circular methods (B and C) is about five times more precise than the average yield obtained using method $\mathrm{A}$. It is to be noted that there is practically no difference between the estimates obtained using method $\mathrm{B}$ and method $\mathrm{C}$ as evident from difference of the estimates obtained from the same area. Moreover, method B is operationally more laborious and time consuming than method C. Results clearly reveal the superiority of circular methods ( $B$ and $\mathrm{C}$ ) over the rectangular method (A) and a similar result was reported by Amin et al. (1994).

The results suggest that method $C$ i.e., a single circular cut where the plants falling inside the circumference of the circle are harvested, may be followed for crop-cut in estimating transplanted rice yield. It is suggested to make at least two cuts in each plot to minimize the chance of over or under estimation of the whole plot yield and the radius of the circle should be $178.5 \mathrm{~cm}$ so that the cut area becomes $10 \mathrm{~m}^{2}$ in order to easy conversion of the crop-cut yield to tha-1.

\section{CONCLUSIONS}

A detail block-wise account of area and the number of plots planted to rice crop in different seasons has to be made at every fiveyear interval.

In each year, a detail block-wise account of the number of plots planted to rice crop in different seasons has to be made.

Three crop-cuts have to be made in three rice fields for each variety under each land type. 
Table 1. Yield $\left(\mathrm{t} \mathrm{ha}^{-1}\right)$ of transplanted rice using different crop-cut methods in Boro season.

\begin{tabular}{lcccc}
\hline Cut no. & \multicolumn{4}{c}{ Yield $\left(\mathrm{t} \mathrm{ha}^{-1}\right)$} \\
\cline { 2 - 5 } & Method A & Method B & Method C & Difference between methods B and C \\
\hline 1 & 6.97 & 6.20 & 6.21 & -0.01 \\
2 & 6.73 & 6.63 & 6.63 & 0.00 \\
3 & 6.41 & 6.06 & 6.03 & 0.02 \\
4 & 6.60 & 6.29 & 6.31 & -0.02 \\
5 & 7.16 & 6.08 & 6.09 & -0.01 \\
6 & 5.54 & 5.89 & 5.89 & 0.00 \\
Mean & 6.57 & 6.19 & 6.21 & \\
Standard deviation & 0.58 & 0.26 & 0.26 & \\
Relative Precision $(\%)$ & 100 & 522 & 510 & \\
Plot yield $\left(\mathrm{t} \mathrm{ha}^{-1}\right)=6.30$ & & & & \\
\hline
\end{tabular}

A single circular cut where the plants falling inside the circumference of the circle are to be harvested for crop-cut in estimating transplanted as well as direct seeded rice yield and the radius of the circle should be $178.5 \mathrm{~cm}$ so that the cut area becomes $10 \mathrm{~m}^{2}$ in order to easy conversion of the crop-cut yield to real yield at $14 \%$ moisture.

At least two crop-cuts have to be made in each plot to minimize the chance of over or under estimation of the rice yield.

DAE collects the information at the block in collaboration with BBS at upazila level. Upazila agriculture office will lead the coordination of the activities of DAE and BBS personnel.

\section{REFERENCES}

Amin, MK D, D N R Paul and K Rahim. 1994. Evaluation of five crop-cut methods for estimating transplanted rice yield. Bangladesh J. Agri. 19 (14).
Amin, MK D, DN R Paul and K Rahim. 1998. Evaluation of sample-hill method as an alternative to cropcut method for estimating transplanted rice yield. Bangladesh Rice Journal, Vol. IX, No. 1 and 2, P: 39-43.

BBS. 1982. Agricultural Year Book of Bangladesh. pp 14.

BBS. 2013. Report on uniform BBS-DAE crop cutting methods for assessing rice production in last Boro season.

BBS. 2014. Report on recent experience of crop cutting experiments in Bangladesh for annual yield estimation of rice.

DAE. 1990. Report on crop-cut area estimation in Bangladesh.

Mahalanobis, P C. 1945. A Report on Bihar Crop Survey, 1943-44, Sankhya, 7,29-118.

Murthy, C S. 1997. Design of crop cutting experiments with satellite data for crop yield estimation in irrigated command areas. Geocarto International 12(2):5-11.

Sukhatme, P V and V G Panse. 1951. Crop surveys in India-II. Jour. Ind. Soc. Agril. Statist.Vol.III:(2), 95168. 